\title{
Estudio de sequías meteorológicas anuales por medio del índice de aridez, en el estado de Zacatecas, México
}

\author{
Annual Meteorological Droughts Study by Means of the Aridity Index, \\ in the Zacatecas State of Mexico
}

\author{
Campos-Aranda Daniel Francisco \\ Profesor Jubilado de la UASLP \\ Correo:campos_aranda@hotmail.com
}

Información del artículo: recibido: septiembre de 2015, aceptado: mayo de 2016

\begin{abstract}
Resumen
Las sequías meteorológicas son una aberración temporal de la ocurrencia normal de la precipitación. Sus características, como: duración, severidad, frecuencia y área afectada, están gobernadas por los parámetros climáticos de la región, que son consecuencia de la dinámica atmosférica global y por ello, su estudio se ha abordado con base en el índice de aridez $(I A=P / E T P)$, definido como el cociente entre la precipitación $(P)$ y la evapotranspiración potencial $(E T P)$, ambas anuales. En este trabajo se analizan estadísticamente las 16 series cronológicas del $I A$, calculadas en el periodo común de 65 años de 1950 a 2014, en el estado de Zacatecas, México; para definir de manera puntual la ocurrencia de las sequías leves, moderadas, severas y extremas. Además, tales registros se integran en tres zonas geográficas y entonces, se analiza la ocurrencia de las sequías severas y extremas por regiones y en dos periodos del lapso común. Se detectan años con mayor número de estaciones climatológicas afectadas y se formulan las conclusiones sobre los años y secuencias de estos, con sequías críticas y libres de tales eventos.
\end{abstract}

\section{Descriptores:}

- sequías meteorológicas anuales

- índice de aridez anual

- precipitación anual

- evapotranspiración potencial anual

- pruebas estadísticas

- promedios móviles 


\begin{abstract}
Meteorological droughts are a temporary aberration of the normal occurrence of precipitation. Their characteristics, such as duration, severity, frequency and affected area, are governed by climatic parameters of the region, which are a consequence of the global atmospheric dynamics and therefore their study has been addressed based on the aridity index $(A I=P / P E T)$, defined as the ratio of precipitation $(P)$ to potential evapotranspiration (PET), both annual. This paper statistically analyzes the 16 time series of the IA, in the state of Zacatecas, Mexico, calculated on the common 65-year period from 1950 to 2014; in order to punctually define the occurrence of light, moderate, severe and extreme droughts. Moreover, such records are integrated into three geographic zones and then, the occurrence of the severe and extreme droughts is analyzed by regions and in the two periods of the common lapse. Years with the most affected number of climatological stations are identified and conclusions on the years and their sequences, with critical droughts and free of such events are formulated.
\end{abstract}
Keywords:
- annual meteorological droughts
- annual aridity index
- annual precipitation
- annual potential evapotranspiration
- statistical tests
- moving averages

\section{Introducción}

\section{Generalidades}

Las sequías son quizás la amenaza natural más compleja. Las sequías son reconocidas como un desastre ambiental que interesa a los meteorólogos, hidrólogos, ecólogos y agrónomos. Ocurriendo prácticamente en todas las zonas climáticas, las sequías también se estudian por ingenieros civiles, economistas y sociólogos. Una sequía es un periodo prolongado del déficit de agua, que ocurre en una zona que no recibe la precipitación normal durante varios meses o algunos años. La sequía es una aberración temporal. En contraste, la aridez es una característica permanente del clima y está restringida a regiones de baja precipitación. Con frecuencia existe confusión, entre onda de calor y sequía, pero el lapso de ocurrencia típico de la primera es de una semana y la segunda persiste por meses o incluso años. La ocurrencia simultánea de ambos fenómenos meteorológicos tiene consecuencias socioeconómicas desastrosas (Morid et al., 2006; Mishra y Singh, 2010).

Según Byun y Wilhite (1999), debido a su complejidad, las sequías se han estudiado con cuatro enfoques; el primero busca mejorar el entendimiento de la circulación atmosférica asociado a su ocurrencia. El segundo intenta evaluar la severidad, frecuencia y extensión de las sequías de diversas duraciones. El tercero se encarga de describir y cuantificar los impactos de las sequías, centrándose en las pérdidas y sus costos. Por último, el cuarto enfoque busca estrategias de preparación y medidas de mitigación de los daños de las sequías.
Los estudios del segundo enfoque, pretenden definir el inicio y final de las sequías, así como su intensidad; se llevan a cabo con base en diversos índices de sequía, los cuales por lo general utilizan datos de precipitación, temperatura, evapotranspiración y el balance de humedad en el suelo. Comenzaron a mediados de los años sesenta (Palmer, 1965) y han evolucionado hasta el presente, ahora intentando pronosticar las sequías (Mishra y Singh, 2011).

Los estudios orientados a caracterizar las sequías de una localidad (Dogan et al., 2012; Campos, 2015a), comúnmente se basan en detectar las anomalías de la precipitación en diversas duraciones y con diversos índices. Por otra parte, los estudios de carácter regional (Tsakiris et al., 2007; Elagib, 2009), se han abordado utilizando índices que emplean tanto el abastecimiento atmosférico (precipitación $P$ ), como la demanda atmosférica (evapotranspiración potencial ETP), por ejemplo a través del índice de aridez (IA), definido como el cociente de ambas estimaciones $(I A=P / E T P)$.

\section{Justificación del estudio}

El objetivo de este trabajo consiste en analizar regionalmente el comportamiento de las sequías anuales en el estado de Zacatecas, México. Lo anterior, a través del índice de aridez, calculado utilizando los registros de 16 estaciones climatológicas de amplio periodo, para el lapso común de 65 años, de 1950 a 2014. De acuerdo con la ubicación en el estado, de los 16 registros, se establecen tres zonas de estudio (norte, centro y sur) y se procesan estadísticamente en ellas los índices de aridez anual, para caracterizar regionalmente los años con sequías severas y extremas. 


\section{Métodos y materiales}

Regiones climáticas e índice de aridez

De manera general, la inconsistencia o irregularidad de las variables que caracterizan los procesos del ciclo hidrológico, es la responsable de la ocurrencia de los eventos extremos y por ello, regiones con alta variabilidad en la lluvia y su escurrimiento, son más propensas a las sequías y las crecientes (Pandey et al., 2008). De acuerdo con Ponce et al. (2000), las regiones centrales del espectro climático, es decir, los climas árido, semiárido y subhúmedo $(0.0833<I A<1.3333)$, son las más susceptibles a desarrollar sequías severas y extremas. También se acepta que el cambio climático puede acrecentar el déficit hídrico, generado por la demanda en aumento debido al desarrollo económico.

En resumen, las características de las sequías están gobernadas por los parámetros climáticos regionales, entre ellos la precipitación y la evapotranspiración, los cuales son relativamente fáciles de estimar. La precipitación de una localidad depende de la latitud, época del año, factores geográficos, proximidad a los océanos, circulación atmosférica de mesoescala, presión atmosférica y condiciones de la superficie terrestre. En cambio, la evapotranspiración depende de la radiación solar neta, del déficit de presión de vapor, de la rugosidad de la superficie y de su índice de área foliar (Ponce et al., 2000; Pandey y Ramasastri, 2002; Pandey et al., 2008).

Tomando en cuenta lo anterior, el United Nations Environment Programme propuso originalmente (UNEP, 1992) el Cociente de Sequedad (dryness ratio) anual para caracterizar las zonas desérticas, cuando tal relación P/ETP es menor de 0.200 . Recientemente, tal cociente se denomina Índice de Aridez (aridity index) IA y define los climas áridos con $0.050<I A<0.200$ y los hiperáridos con $I A<0.050$ (UNEP, 2006).

\section{Registros de precipitación anual}

Al analizar las amplitudes de los 30 registros de lluvia anual procesados por Campos (2015b) en el estado de Zacatecas, México, se observa que 16 de ellos tienen registros mayores de 63 datos hasta el año de 2012. Esas series se seleccionaron. En esa referencia se detalla cómo se integraron los registros y cómo se dedujeron sus valores faltantes. A través de la Dirección Local Zacatecas de la Comisión Nacional del Agua, se obtuvieron los datos correspondientes a los años 2013 y 2014, tanto de precipitación anual como de temperatura media anual. Los 16 registros de las estaciones climatológicas que serán procesados, definen un periodo común máximo de 65 años en el lapso de 1950 a 2014. En la tabla 1 se citan los datos generales y sus respectivos valores promedio anual de precipitación (PMA); en esta tabulación las estaciones se exponen por zonas y en orden progresivo de PMA. En la figura 1 se muestra su ubicación dentro del estado de Zacatecas, el cual se localiza en el centro de México.

\section{Registros de temperatura media anual}

De igual forma a como se integraron los registros de lluvia anual en el estudio de Campos (2015b), se obtuvieron ahora los 16 de temperatura media de cada año. Como algunos de estos registros comenzaron varios años después de los pluviométricos, para tales valores faltantes se empleó su promedio anual. Tal fue el caso, en las estaciones Cañitas de Felipe Pescador, Río Grande, Villa de Cos y Villanueva. En la tabla 1 se dan los valores promedio anual de temperatura media (TMA).

\section{Estimación de evapotranspiración potencial anual}

Para la estimación de la ETP anual se empleó la fórmula empírica de Hargreaves y Samani (1982), la cual permite la estimación de la ETP media diaria en milímetros, con base únicamente en la temperatura media (Tt) expresada en grados Fahrenheit y de la radiación solar incidente media diaria expresada en milímetros de lámina de agua evaporada $\left(R i^{\prime}\right)$, tal ecuación es:

$E T P=0.0075 \cdot R i^{\prime} \cdot T t$

La radiación solar incidente $(R i)$ se aproximó al promedio de los 12 valores mensuales, citados por Campos (2015b), obtenidos para la ciudad de Zacatecas en los mapas de Almanza y López (1975); se adoptó un valor de $495 \mathrm{cal} / \mathrm{cm}^{2} /$ día. Para transformar a lámina de agua evaporada se emplea:

$R i^{\prime}=10 \cdot R i / H v$

en donde, $\mathrm{Hv}$ es el llamado calor latente de evaporación o energía necesaria para evaporar $1 \mathrm{~g}$ o un $\mathrm{cm}^{3}$ de agua, se estima con la ecuación siguiente, estando la temperatura media $(\mathrm{Tt})$ en ${ }^{\circ} \mathrm{C}$ :

$H v=595.9-0.55 \cdot T t$

Utilizando en las ecuaciones 1 y 3 la temperatura media anual y el valor de $R i=495 \mathrm{lg} / \mathrm{d}$ se obtiene la ETP media diaria, que al multiplicarse por 365 conduce al valor buscado de la ETP anual. También en la tabla 1 se muestran los valores promedio anual en los 16 registros procesados. 
Tabla 1. Datos generales de las 16 estaciones climatológicas procesadas del estado de Zacatecas, México

\begin{tabular}{|c|c|c|c|c|c|c|c|c|c|}
\hline Núm. & Estación (Núm. en el AC) & $\begin{array}{l}\text { Latitud } \\
\mathrm{N}\end{array}$ & $\begin{array}{l}\text { Long. } \\
\text { WG }\end{array}$ & $\begin{array}{l}\text { Altitud } \\
\text { (msnm) }\end{array}$ & Zona & $\begin{array}{l}P M A \\
(\mathrm{~mm})\end{array}$ & $\begin{array}{l}\text { TMA } \\
\left({ }^{\circ} \mathrm{C}\right)\end{array}$ & $\begin{array}{c}\text { ETP } \\
(\mathrm{mm})\end{array}$ & $I A$ \\
\hline 1 & Cañitas de Felipe P. (9) & $23^{\circ} 36^{\prime}$ & $102^{\circ} 44^{\prime}$ & 2025 & Norte & 371.3 & 16.0 & 1404.3 & 0.265 \\
\hline 2 & Río Grande (73) & $23^{\circ} 48^{\prime}$ & $103^{\circ} 02^{\prime}$ & 1890 & Norte & 384.7 & 16.9 & 1442.7 & 0.267 \\
\hline 3 & Fresnillo (30) & $23^{\circ} 11^{\prime}$ & $102^{\circ} 53^{\prime}$ & 2195 & Norte & 415.9 & 16.9 & 1442.4 & 0.289 \\
\hline 4 & Leobardo Reynoso (25) & $23^{\circ} 11^{\prime}$ & $103^{\circ} 12^{\prime}$ & 2090 & Norte & 418.3 & 16.1 & 1406.9 & 0.298 \\
\hline 5 & Villa de Cos (98) & $23^{\circ} 17^{\prime}$ & $102^{\circ} 21^{\prime}$ & 2050 & Norte & 426.4 & 17.5 & 1468.2 & 0.291 \\
\hline 6 & Santa Rosa (69) & $22^{\circ} 56^{\prime}$ & $103^{\circ} 07^{\prime}$ & 2240 & Norte & 459.3 & 14.8 & 1349.8 & 0.341 \\
\hline 7 & San Pedro Piedra G. (83) & $22^{\circ} 27^{\prime}$ & $102^{\circ} 21^{\prime}$ & 2032 & Centro & 411.5 & 16.8 & 1439.7 & 0.286 \\
\hline 8 & Villa García (99) & $22^{\circ} 10^{\prime}$ & $101^{\circ} 57^{\prime}$ & 2102 & Centro & 443.3 & 16.3 & 1417.6 & 0.313 \\
\hline 9 & Pinos (65) & $22^{\circ} 17^{\prime}$ & $101^{\circ} 35^{\prime}$ & 2408 & Centro & 448.3 & 16.3 & 1415.9 & 0.318 \\
\hline 10 & Zacatecas (103) & $22^{\circ} 46^{\prime}$ & $102^{\circ} 35^{\prime}$ & 2485 & Centro & 463.2 & 15.7 & 1390.6 & 0.334 \\
\hline 11 & Villanueva (102) & $22^{\circ} 22^{\prime}$ & $102^{\circ} 53^{\prime}$ & 1920 & Centro & 470.9 & 16.9 & 1441.3 & 0.327 \\
\hline 12 & Presa El Chique (68) & $22^{\circ} 00^{\prime}$ & $102^{\circ} 53^{\prime}$ & 1620 & Sur & 543.6 & 20.9 & 1615.0 & 0.337 \\
\hline 13 & Juchipila (42) & $21^{\circ} 23^{\prime}$ & $103^{\circ} 07^{\prime}$ & 1270 & Sur & 691.7 & 21.7 & 1649.7 & 0.419 \\
\hline 14 & Nochistlán (58) & $21^{\circ} 22^{\prime}$ & $102^{\circ} 51^{\prime}$ & 1850 & Sur & 700.2 & 18.6 & 1515.4 & 0.462 \\
\hline 15 & Tlaltenango (94) & $21^{\circ} 47^{\prime}$ & $103^{\circ} 18^{\prime}$ & 1700 & Sur & 701.5 & 18.0 & 1491.4 & 0.471 \\
\hline 16 & Excamé (27) & $21^{\circ} 39^{\prime}$ & $103^{\circ} 20^{\prime}$ & 1740 & Sur & 736.5 & 18.5 & 1512.0 & 0.488 \\
\hline
\end{tabular}

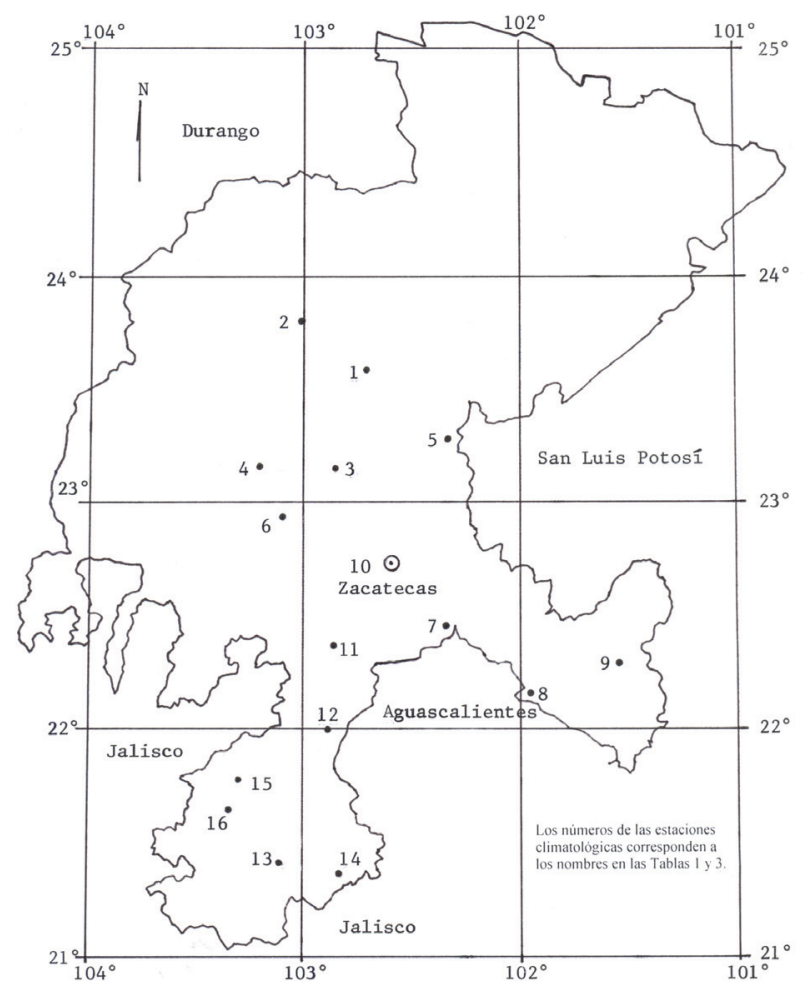

Figura 1. Ubicación de las 16 estaciones climatológicas procesadas del estado de Zacatecas, México

\section{Cálculo de las series de índices de aridez}

Al dividir los valores de la precipitación anual entre los de la ETP anual calculados en cada uno de los 16 registros procesados, se definen las 16 series de 65 datos de índices de aridez $(I A)$, cuyos valores promedio anual se tienen en la tabla 1. En cambio, en la tabla 2 se exponen los datos y resultados anuales, en los tres registros seleccionados como representativos de cada zona geográfica analizada. En la figura 2 se muestran dos de tales registros de $I A$, como series cronológicas.

Determinación del tipo de sequía

Cada serie de $I A$ anuales se ordena de menor a mayor. En seguida se obtiene su mediana y se considera que todos los IA menores que ella son sequías; con esta mitad del registro, se forman cuatro grupos buscando que tengan igual número de elementos $(n s)$. Los $n s$ valores más bajos definen los años con sequía extrema (SE), los siguientes $n s$ elementos establecen los años con sequía severa (SS), después habrá $n s$ valores de $I A$ relativos a los años con sequía moderada (SM) y por último, $n s$ magnitudes de $I A$ que terminan antes de la mediana y que son años con sequía leve o ligera (SL). En las columnas 7, 12 y 17 de la tabla 2 se tienen las designaciones del tipo de sequía (TS), obtenidas con el procedimiento anterior usando $n s=8$, en los tres registros representativos de las zonas analizadas del estado de Zacatecas, México. Cuando se conozcan los porcentajes de cada tipo de sequía que ocurren en la localidad o región estudiada, se aplicarán a la mitad del registro y entonces el $n s$ será variable.

\section{Análisis estadísticos y regionales}

Verificación de la homogeneidad

Con base en cada serie cronológica de $I A$ anual se realizó un análisis de calidad estadística, buscando compo- 


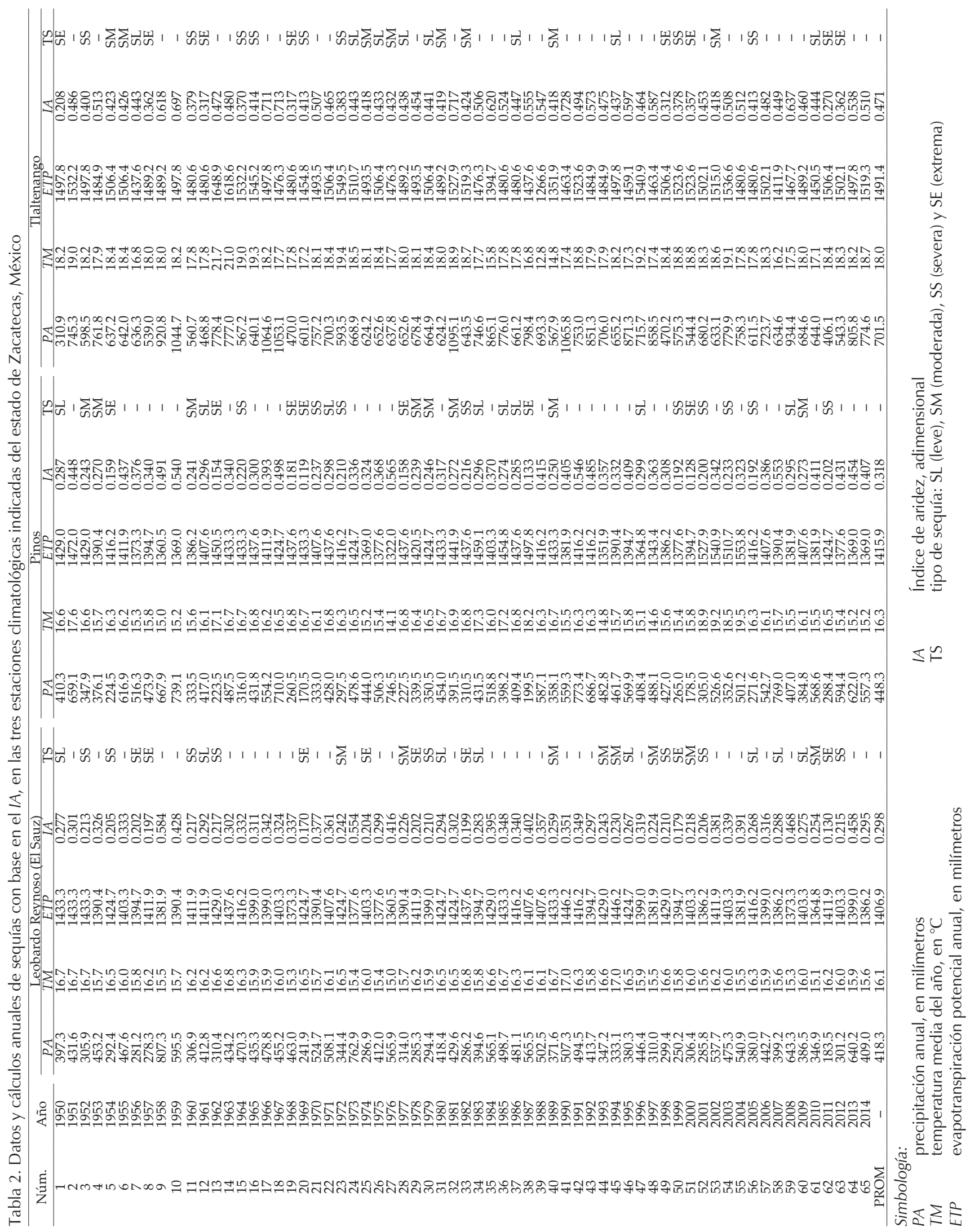



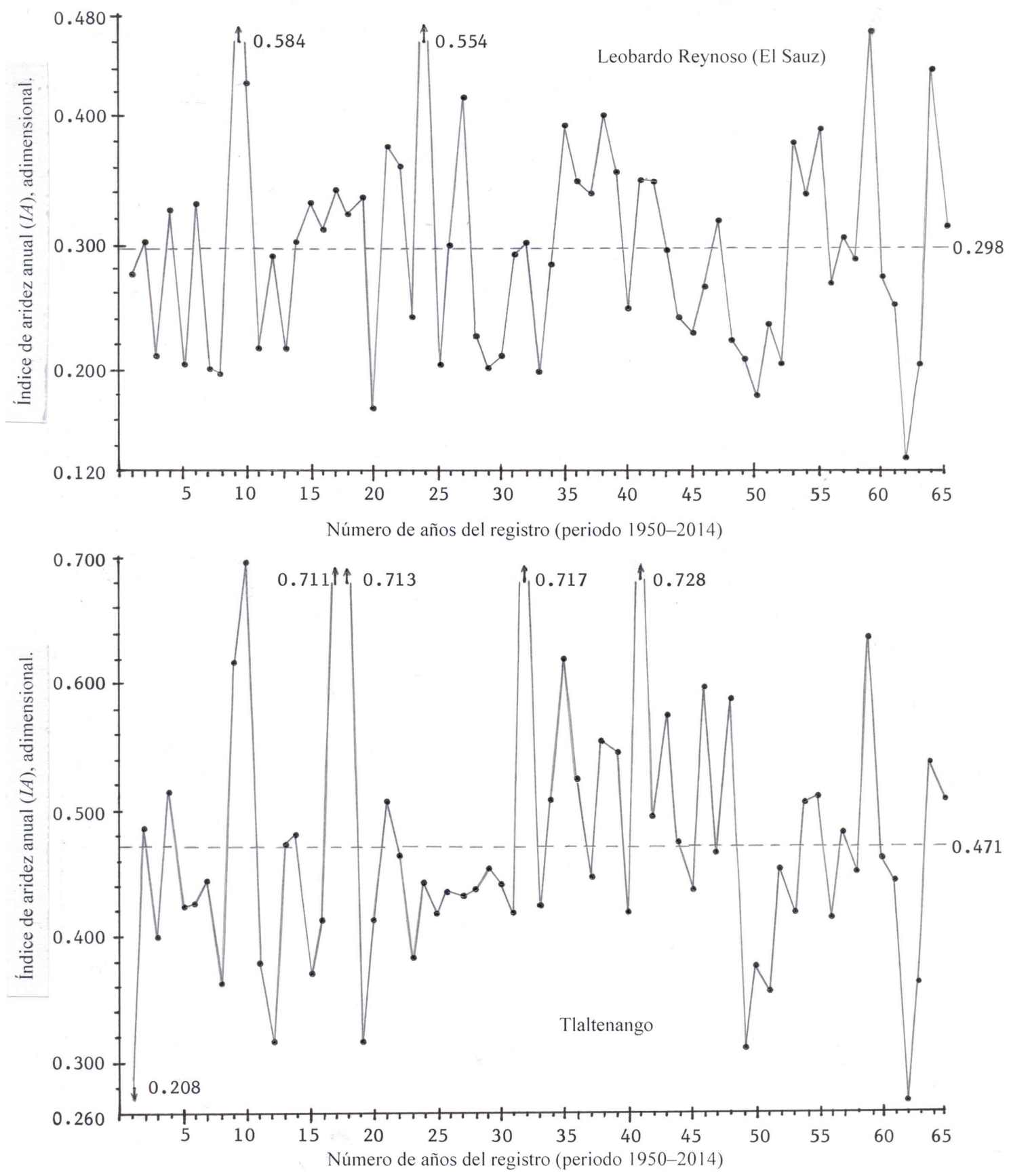

Figura 2. Series cronológicas del índice de aridez en las estaciones climatológicas Leobardo Reynoso (El Sauz) y Tlaltenango, del estado de Zacatecas, México

nentes determinísticas con dos pruebas generales (test de Helmert y Secuencias) y seis específicas: de persistencia (Anderson y Sneyers), de tendencia (Kendall y Spearman), de variabilidad (Bartlett) y de cambio en la media (Cramer). La mayoría de estas pruebas se pueden consultar en WMO (1971), o en Machiwal y Jha (2008; 2012). En la tabla 3 se concentran los resultados de tales pruebas; observándose que 11 registros son perfectamente homogéneos, cuatro muestran persistencia y uno alta variabilidad. Se detecta de la tabla 3, que la persistencia está asociada al valor de coeficiente de correlación serial de orden uno $\left(r_{1}\right)$. Es relevante indicar que en el lapso de estudio (1950-2014), ningún registro de $I A$ presentó tendencia. 
Tabla 3. Coeficiente de correlación serial de orden uno $\left(r_{1}\right)$ y resultados de las pruebas de homogeneidad, en las 16 estaciones climatológicas procesadas del estado de Zacatecas

\begin{tabular}{clrl}
\hline Núm. & \multicolumn{1}{c}{ Estación } & \multicolumn{1}{c}{$r_{1}$} & \multicolumn{1}{c}{ Resultados de las pruebas de homogeneidad } \\
\hline 1 & Cañitas de Felipe P. & -0.129 & Homogénea \\
2 & Río Grande & -0.075 & Según pruebas básicas (Helmert y Secuencias) oscila mucho \\
3 & Fresnillo & 0.034 & Homogénea \\
4 & Leobardo Reynoso & 0.013 & Homogénea \\
5 & Villa de Cos & 0.012 & Homogénea \\
6 & Santa Rosa & 0.176 & Según pruebas básicas oscila poco y muestra persistencia ligera \\
7 & San Pedro Piedra G. & 0.133 & Homogénea \\
8 & Villa García & -0.031 & Homogénea \\
9 & Pinos & 0.160 & Oscila poco según test de Helmert \\
10 & Zacatecas & 0.097 & Homogénea \\
11 & Villanueva & 0.388 & Según pruebas básicas oscila poco y se detecta persistencia \\
12 & Presa El Chique & 0.174 & Según pruebas básicas oscila poco y muestra persistencia ligera \\
13 & Juchipila & 0.093 & Homogénea \\
14 & Nochistlán & 0.047 & Homogénea \\
15 & Tlaltenango & 0.052 & Homogénea \\
16 & Excamé & 0.092 & Homogénea \\
\hline
\end{tabular}

Los diez años más secos

A partir de las series de valores $I A$ ordenados de menor a mayor, se seleccionan los diez más bajos y se obtienen sus años de ocurrencia. Tales valores de $I A$ y sus años respectivos se han concentrado en la tabla 4. Para obtener en cuáles años se presentó el mayor número de sequías más extremas, se realizó una tabulación por zona geográfica, con 65 renglones relativos a cada año del periodo analizado (1950-2014) y diez columnas para los órdenes de magnitud decrecientes $(10,9, \ldots, 2,1)$.

Después cada uno de los diez años de cada registro (tabla 4), se llevaron a la tabulación de su zona, marcando únicamente su ocurrencia en el renglón y columna que le corresponde; se sumaron los respectivos órdenes de magnitud y se designaron por SUM. Los años con 3 o más ocurrencias, es decir, estaciones climatológicas afectadas (ECA) con sequía extrema, se exponen en la tabla 5.

En la tabla 5 se observa que en las zonas norte, centro y sur ocurren, respectivamente, 10,9 y 8 años con tres o más estaciones climatológicas afectadas. En un contexto secuencial, los 18 años detectados en la Tabla 5 son los siguientes: 1950, 1952, 1954, 1956, 1957, 1960, 1961, 1962, 1969, 1974, 1979, 1982, 1989, 1998, 1999, 2000, 2001 y 2011; mostrando que las sequías extremas en el estado de Zacatecas, México, tienen un intervalo promedio de recurrencia de aproximadamente 10 años. También se observa que las sequías extremas de los años cincuenta fueron las de más amplia duración y cobertura. Se deduce de la Tabla 5, que los años con sequías extremas en las tres zonas geográficas fueron exclusivamente, 1957, 1999 y 2011.
Secuencias mínimas de 5 años

Con base en la técnica de promedios móviles de orden cinco, se identificaron en las 16 series cronológicas del $I A$, las tres secuencias mínimas, cuyos valores y ubicación se detallan en la tabla 6 . Se deduce que en la zona Norte las secuencias de 5 años con sequías más severas son de 1997 al 2001, después la de 1950 a 1957 y por último, según la estación Fresnillo, la de 1960 a 1965. En la zona Centro y con excepción de la estación Pinos, el resto define un lapso amplio de 1977 a 1989 para estas secuencias mínimas. En la zona Sur, con excepción de Nochistlán, el resto define sus secuencias mínimas de 5 años en el inicio de los registros, es decir, de 1950 a 1957.

\section{Estudio regional de las sequías severas y extremas}

En la tabla 7 se indican únicamente los años con sequía severa (SS) y extrema (SE); está dividida en dos periodos, del inicio a 1980 y de 1981 al 2014. La suma de tales sequías en las zona Norte, Centro y Sur es, respectivamente, de 56, 43 y 41 eventos en el primer periodo estudiado y de 40, 37 y 39 eventos, en el segundo lapso de análisis. En resumen, en el primer periodo estudiado ocurrió un total de 140 eventos (SS y SE) y en el segundo lapso, únicamente 116 eventos.

Contrario a lo que se podría esperar debido a los efectos del cambio climático, no ocurren más sequías extremas en las épocas recientes, es decir, en el lapso de 1981 a 2014, en comparación con el periodo de 1950 a 1980. Para reforzar lo anterior, se observa en la última columna de la tabla 7, que el número de años sin se- 
Tabla 4. Valores de los 10 menores índices de aridez (IA) anuales y sus años respectivos, en las 16 estaciones climatológicas procesadas del estado de Zacatecas, México

\begin{tabular}{|c|c|c|c|c|c|c|c|c|c|c|c|c|}
\hline \multirow[b]{2}{*}{ Núm. } & \multirow[b]{2}{*}{ Nombre } & \multirow[b]{2}{*}{ Dato } & \multicolumn{10}{|c|}{ Valores de $I A$ en orden creciente de magnitud y sus años respectivos } \\
\hline & & & 10 & 9 & 8 & 7 & 6 & 5 & 4 & 3 & 2 & 1 \\
\hline \multirow[t]{2}{*}{1} & Cañitas de Felipe P. & $I A$ & 0.112 & 0.130 & 0.131 & 0.136 & 0.140 & 0.142 & 0.145 & 0.152 & 0.161 & 0.166 \\
\hline & & Año & 2011 & 1999 & 1980 & 1975 & 1974 & 1952 & 1957 & 2001 & 1969 & 1989 \\
\hline \multirow[t]{2}{*}{2} & Río Grande & $I A$ & 0.090 & 0.101 & 0.107 & 0.138 & 0.152 & 0.160 & 0.162 & 0.168 & 0.173 & 0.174 \\
\hline & & Año & 1957 & 1956 & 1960 & 2011 & 1950 & 1982 & 1995 & 1989 & 2012 & 2001 \\
\hline \multirow[t]{2}{*}{3} & Fresnillo & $I A$ & 0.133 & 0.137 & 0.152 & 0.158 & 0.169 & 0.176 & 0.183 & 0.188 & 0.191 & 0.200 \\
\hline & & Año & 1965 & 2011 & 2012 & 1979 & 1960 & 1964 & 1969 & 1999 & 1974 & 1954 \\
\hline \multirow[t]{2}{*}{4} & Leobardo Reynoso & $I A$ & 0.130 & 0.170 & 0.179 & 0.197 & 0.199 & 0.202 & 0.202 & 0.204 & 0.205 & 0.206 \\
\hline & & Año & 2011 & 1969 & 1999 & 1957 & 1982 & 1956 & 1978 & 1974 & 1954 & 2001 \\
\hline \multirow[t]{2}{*}{5} & Villa de Cos & $I A$ & 0.075 & 0.122 & 0.136 & 0.144 & 0.155 & 0.176 & 0.176 & 0.180 & 0.182 & 0.183 \\
\hline & & Año & 1954 & 1979 & 2011 & 1982 & 1956 & 1999 & 2000 & 1995 & 1963 & 1980 \\
\hline \multirow[t]{2}{*}{6} & Santa Rosa & $I A$ & 0.151 & 0.158 & 0.168 & 0.188 & 0.192 & 0.202 & 0.209 & 0.213 & 0.213 & 0.224 \\
\hline & & Año & 1952 & 1957 & 1950 & 1979 & 2011 & 1953 & 1964 & 1956 & 1969 & 1954 \\
\hline \multirow[t]{2}{*}{7} & San Pedro Piedra G. & $I A$ & 0.080 & 0.122 & 0.127 & 0.129 & 0.139 & 0.162 & 0.170 & 0.172 & 0.174 & 0.180 \\
\hline & & Año & 1988 & 1979 & 1987 & 1969 & 1999 & 1982 & 1960 & 2011 & 1952 & 1950 \\
\hline \multirow[t]{2}{*}{8} & Villa García & $I A$ & 0.147 & 0.162 & 0.168 & 0.176 & 0.177 & 0.183 & 0.189 & 0.192 & 0.198 & 0.207 \\
\hline & & Año & 1969 & 1999 & 1954 & 1989 & 1982 & 1962 & 1974 & 1993 & 1957 & 2000 \\
\hline \multirow[t]{2}{*}{9} & Pinos & $I A$ & 0.119 & 0.128 & 0.133 & 0.154 & 0.158 & 0.159 & 0.181 & 0.192 & 0.192 & 0.200 \\
\hline & & Año & 1969 & 2000 & 1987 & 1962 & 1977 & 1954 & 1968 & 1999 & 2005 & 2001 \\
\hline \multirow[t]{2}{*}{10} & Zacatecas & $I A$ & 0.124 & 0.157 & 0.176 & 0.177 & 0.201 & 0.202 & 0.212 & 0.215 & 0.216 & 0.227 \\
\hline & & Año & 1969 & 2012 & 1957 & 2011 & 1954 & 1978 & 1982 & 1962 & 1979 & 1960 \\
\hline \multirow[t]{2}{*}{11} & Villanueva & $I A$ & 0.147 & 0.154 & 0.155 & 0.173 & 0.185 & 0.188 & 0.192 & 0.194 & 0.213 & 0.214 \\
\hline & & Año & 1960 & 1979 & 1978 & 1969 & 2011 & 1982 & 1952 & 1957 & 1998 & 1961 \\
\hline \multirow[t]{2}{*}{12} & Presa El Chique & $I A$ & 0.170 & 0.190 & 0.195 & 0.223 & 0.240 & 0.240 & 0.244 & 0.248 & 0.249 & 0.258 \\
\hline & & Año & 2011 & 1953 & 1961 & 1957 & 1998 & 1976 & 1989 & 1952 & 1979 & 1997 \\
\hline \multirow[t]{2}{*}{13} & Juchipila & $I A$ & 0.171 & 0.211 & 0.222 & 0.296 & 0.302 & 0.313 & 0.321 & 0.323 & 0.333 & 0.349 \\
\hline & & Año & 2011 & 1957 & 1994 & 2000 & 1956 & 1952 & 1972 & 1950 & 1951 & 2012 \\
\hline \multirow[t]{2}{*}{14} & Tlaltenango & $I A$ & 0.208 & 0.270 & 0.312 & 0.317 & 0.317 & 0.357 & 0.362 & 0.362 & 0.370 & 0.378 \\
\hline & & Año & 1950 & 2011 & 1998 & 1961 & 1968 & 2000 & 2012 & 1957 & 1964 & 1999 \\
\hline \multirow[t]{2}{*}{15} & Nochistlán & $I A$ & 0.219 & 0.262 & 0.265 & 0.278 & 0.281 & 0.314 & 0.321 & 0.322 & 0.325 & 0.341 \\
\hline & & Año & 1989 & 1952 & 2005 & 2001 & 1999 & 1960 & 2011 & 1959 & 1998 & 2009 \\
\hline \multirow[t]{2}{*}{16} & Excamé & $I A$ & 0.230 & 0.265 & 0.280 & 0.310 & 0.336 & 0.336 & 0.347 & 0.360 & 0.377 & 0.378 \\
\hline & & Año & 2011 & 1957 & 1994 & 1993 & 2000 & 1969 & 1961 & 1998 & 1999 & 1950 \\
\hline
\end{tabular}

Tabla 5. Años con tres o más estaciones climatológicas afectadas (ECA), por zonas geográficas en el estado de Zacatecas, México

\begin{tabular}{ccccccccc}
\hline \multicolumn{3}{c}{ Zona Norte $(\mathrm{EC}=6)$} & \multicolumn{3}{c}{ Zona Centro $(\mathrm{EC}=5)$} & \multicolumn{3}{c}{ Zona Sur $(\mathrm{EC}=5)$} \\
\hline Año & ECA & SUM & Año & ECA & SUM & Año & ECA & SUM \\
\hline 1954 & 4 & 14 & 1954 & 3 & 19 & 1950 & 3 & 14 \\
1956 & 4 & 23 & 1957 & 3 & 13 & 1952 & 3 & 17 \\
1957 & 4 & 30 & 1960 & 3 & 15 & 1957 & 4 & 28 \\
1969 & 4 & 17 & 1962 & 3 & 15 & 1961 & 3 & 19 \\
1974 & 3 & 13 & 1969 & 5 & 44 & 1998 & 4 & 19 \\
1979 & 3 & 23 & 1979 & 3 & 20 & 1999 & 3 & 9 \\
1982 & 3 & 18 & 1982 & 4 & 20 & 2000 & 3 & 18 \\
1999 & 3 & 20 & 1999 & 3 & 18 & 2011 & 5 & 43 \\
2001 & 3 & 5 & 2011 & 3 & 16 & - & - & - \\
2011 & 7 & 58 & - & - & - & - & - & - \\
\hline
\end{tabular}


DOI: https://doi.org/10.1016/j.riit.2016.07.010

Campos-Aranda Daniel Francisco

Tabla 6. Promedios y lapsos de las tres secuencias mínimas de cinco años en las series cronológicas del $I A$, en las 16 estaciones climatológicas procesadas del estado de Zacatecas, México

\begin{tabular}{clccc}
\hline Núm. & \multicolumn{1}{c}{ Estación } & Primera secuencia & Segunda secuencia & Tercera secuencia \\
\hline 1 & Cañitas de Felipe P. & $0.194(1997-2001)$ & $0.200(2008-2012)$ & $0.202(2007-2011)$ \\
2 & Río Grande & $0.189(1953-1957)$ & $0.208(1952-1956)$ & $0.216(1997-2001)$ \\
3 & Fresnillo & $0.205(1961-1965)$ & $0.212(1960-1964)$ & $0.218(1997-2001)$ \\
4 & Leobardo Reynoso & $0.207(1997-2001)$ & $0.230(1996-2000)$ & $0.239(1998-2002)$ \\
5 & Villa de Cos & $0.187(1997-2001)$ & $0.191(1996-2000)$ & $0.192(1995-1999)$ \\
6 & Santa Rosa & $0.214(1950-1954)$ & $0.229(1952-1956)$ & $0.231(1953-1957)$ \\
7 & San Pedro Piedra G. & $0.206(1985-1989)$ & $0.216(1984-1988)$ & $0.218(1950-1954)$ \\
8 & Villa García & $0.238(1950-1954)$ & $0.255(1985-1989)$ & $0.267(1996-2000)$ \\
9 & Pinos & $0.209(1968-1972)$ & $0.219(1999-2003)$ & $0.234(1998-2002)$ \\
10 & Zacatecas & $0.246(1960-1964)$ & $0.250(1959-1963)$ & $0.266(1978-1982)$ \\
11 & Villanueva & $0.190(1978-1982)$ & $0.212(1977-1981)$ & $0.216(1979-1983)$ \\
12 & Presa El Chique & $0.255(1953-1957)$ & $0.260(1952-1956)$ & $0.260(1951-1955)$ \\
13 & Juchipila & $0.344(1953-1957)$ & $0.344(2009-2013)$ & $0.348(1950-1954)$ \\
14 & Tlaltenango & $0.384(1998-2002)$ & $0.404(1960-1964)$ & $0.406(1950-1954)$ \\
15 & Nochistlán & $0.357(1997-2001)$ & $0.369(1998-2002)$ & $0.379(1996-2000)$ \\
16 & Excamé & $0.403(1998-2002)$ & $0.411(1950-1954)$ & $0.420(2007-2011)$ \\
\hline
\end{tabular}




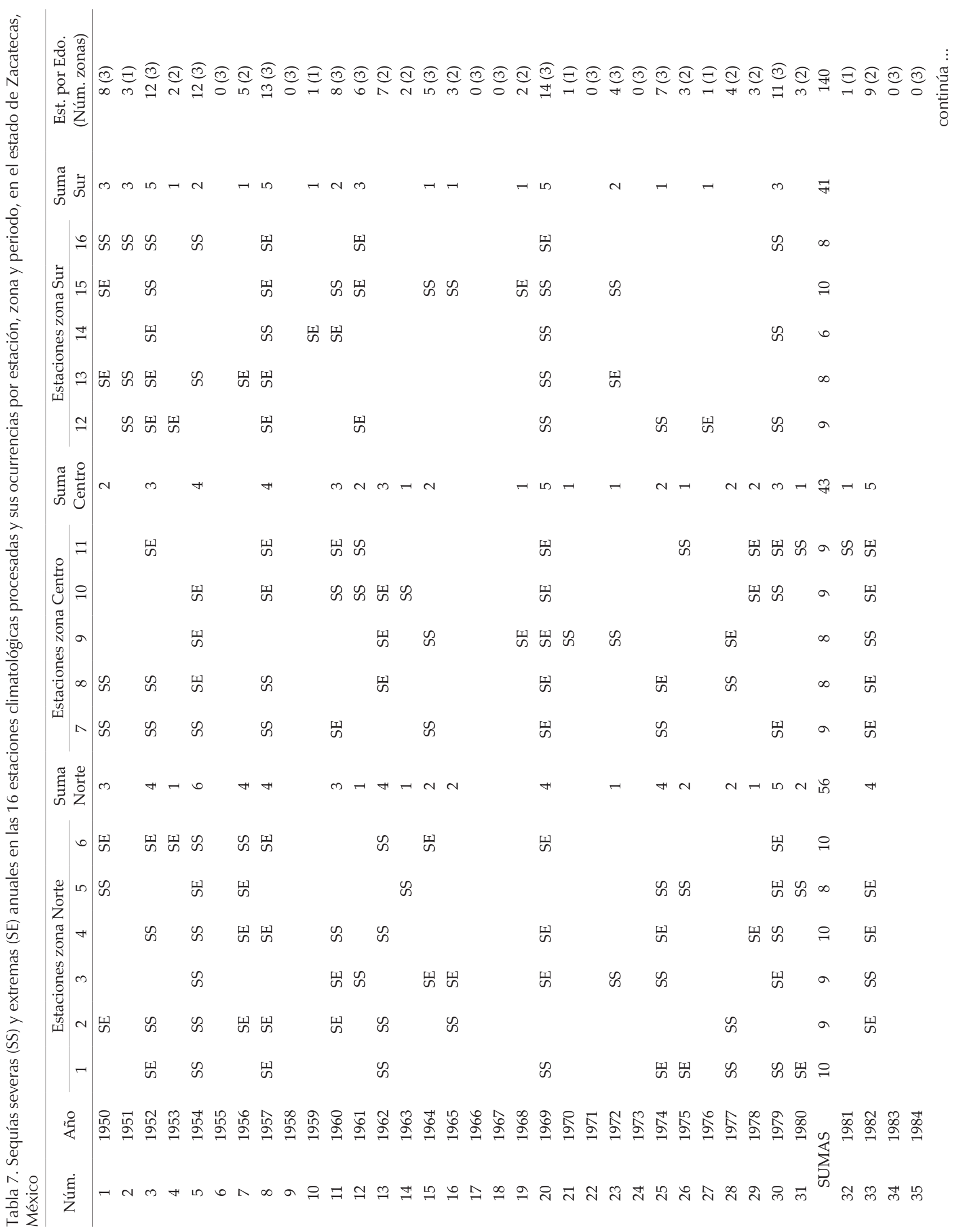




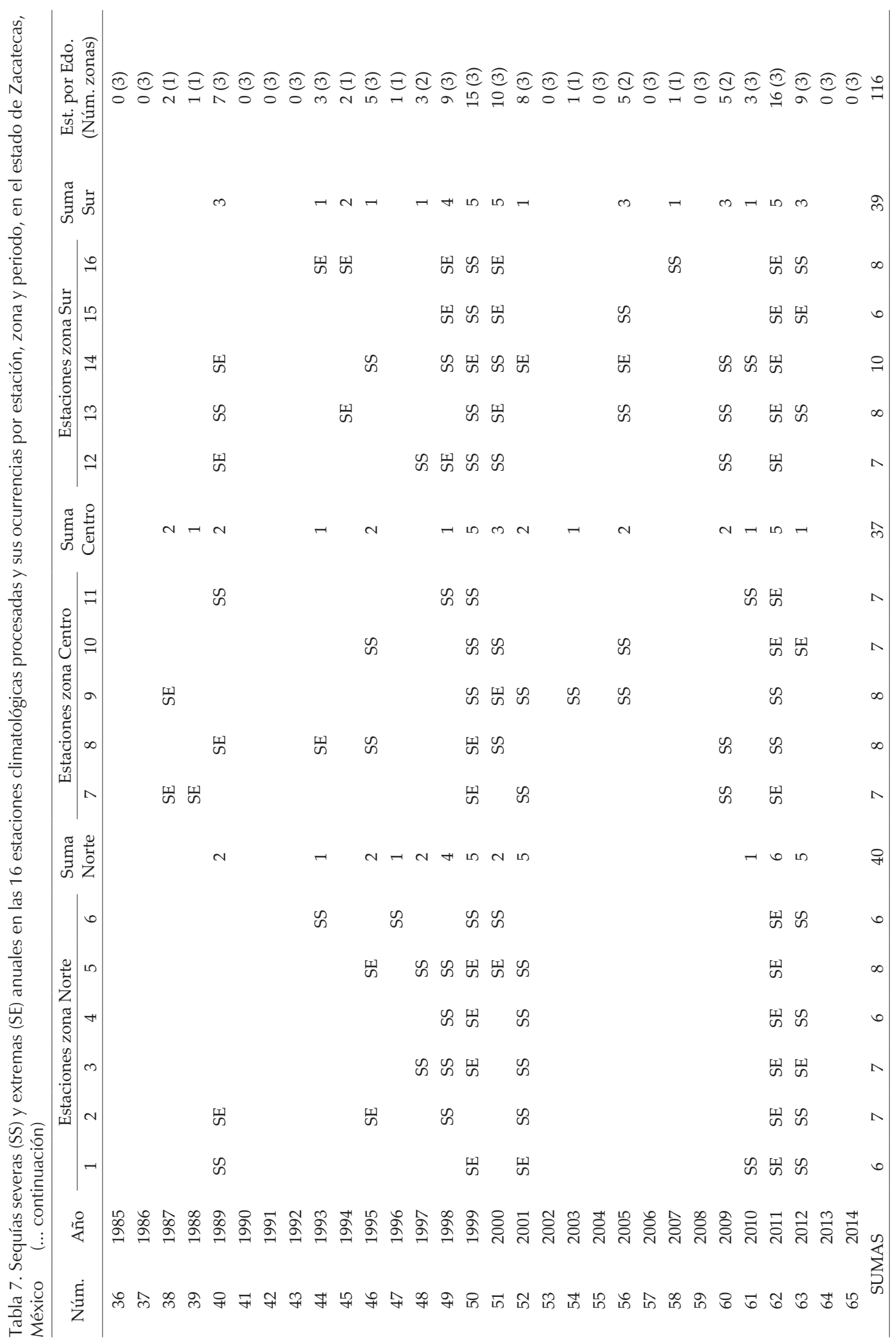


quías extremas (SS y SE) en el primer periodo es de solo $6(1955,1958,1966,1967,1971$ y 1973) y en el segundo de 13, es decir del doble, ocurriendo en este lapso dos secuencias de años sin sequías extremas: 1983 a 1986 y 1990 a 1992.

También se observa en la última columna de la tabla 7, que los años con mayor número de estaciones climatológicas afectadas por las sequías extremas (SS y SE), fueron 1950 con 8, 1952 y 1954 con 12, 1957 con 13, 1960 a 1962 con 6 a 8, 1969 con 14, 1974 con 7, 1979 con 11, 1982 con 9, 1989 con 7, 1998 con 9, 1999 con 15, 2000 con 10, 2001 con 8, 2011 con 16 y 2012 con 9. De los años anteriores, exclusivamente los años de 1962 y 1982 no afectaron la zona Sur. Las sequías de los años 1952, 1954, 1957, 1969 y 1999, abarcaron casi la totalidad de las tres zonas estudiadas. El año 2011 ocurrió en las 16 estaciones climatológicas procesadas.

\section{Conclusiones}

Se ha desarrollado un procedimiento para obtener los cuatro tipos de sequías meteorológicas (leves, moderadas, severas y extremas), en las 16 series cronológicas de índices de aridez anuales $(I A)$ procesadas del estado de Zacatecas, México, lo cual constituye un análisis local o puntual de las sequías. Esas series tuvieron un periodo común de 65 años en el lapso de 1950 a 2014. Los resultados de tal procedimiento se ilustraron en la tabla 2.

El análisis regional, en tres zonas geográficas del estado de Zacatecas, México, permite concluir en relación con las 10 sequías más críticas (tabla 4), que los años con tres o más estaciones climatológicas afectadas por zona fueron: 1950, 1952, 1954, 1956, 1957, 1960, 1961, 1962, 1969, 1974, 1979, 1982, 1989, 1998, 1999, 2000, 2001 у 2011. Únicamente los años 1957, 1999 y 2011 fueron comunes en las tres zonas (Tabla 5).

La presencia generalizada (tabla 6) de las secuencias mínimas de 5 años del $I A$, obtenidas a través de la técnica de promedios móviles, ocurre en la zona norte en el periodo 1997 a 2001, en la zona centro en el lapso de 1977 a 1989 y en la zona sur, en el inicio de los registros de 1950 a 1957.

La ocurrencia global de las sequías severas (SS) y extremas (SE) se analizó en dos periodos, el primero del año 1950 a 1980 y el segundo de 1981 a 2014 (tabla 7). La suma de esas sequías en las zona Norte, Centro y Sur es, respectivamente, de 56, 43 y 41 eventos en el primer periodo estudiado y de 40, 37 y 39 eventos, en el segundo lapso de análisis. Entonces, en el periodo inicial ocurrió un total de 140 eventos y en el segundo, únicamente 116 eventos. Además se observa que el número de años sin sequías críticas (SS y SE), en el primer periodo es de sólo 6 y en el segundo de 13, es decir del doble. Por lo anterior, se concluye que no se detectan efectos del cambio climático en la ocurrencia de sequías críticas en el estado de Zacatecas, México, con los análisis realizados.

Según resultados de la última columna de la tabla 7 , las sequías críticas (SS y SE) de los años 1952, 1954, 1957, 1969, 1979 y 1999, abarcaron casi la totalidad de las tres zonas estudiadas. El año 2011 ocurrió en las 16 estaciones climatológicas procesadas. Con base en estos resultados y de manera aproximada, se deduce que las sequías críticas en el estado de Zacatecas, México, tienen una recurrencia del orden de los 10 años.

Todos los análisis estadísticos realizados con los $I A$ anuales, se pueden llevar a cabo con el mes o meses más lluviosos, así como para la época de lluvias normal, como lo ha ilustrado Elagib (2009).

\section{Agradecimientos}

Se aprecian las observaciones y correcciones sugeridas por los dos árbitros anónimos, las cuales permitieron mejorar la redacción del texto y clarificar sus resultados y conclusiones.

\section{Referencias}

Almanza R. y López S. Radiación solar global en la República Mexicana mediante datos de insolación. Series del Instituto de Ingeniería, Núm. 357, UNAM, Ciudad Universitaria, México, 1975, 20 p.

Byun H.R. y Wilhite D.A. Objetive quantification of drought severity and duration. Journal of Climate, volumen 12 (número 9), 1999: 2747-2756.

Campos Aranda D.F. Contraste de los índices DPP, SPI y RDI para clasificación de Sequías, en la estación climatológica Zacatecas, México. Tecnología y Ciencias del Agua, volumen VI (número 1), 2015a: 183-193.

Campos Aranda D.F. Búsqueda de tendencias en la precipitación anual del estado de Zacatecas, México; en 30 registros con más de 50 años. Ingeniería. Investigación y Tecnología, Volumen XVI (número 3), 2015b: 357-370.

Dogan S., Berktay A., Singh V.P. Comparison of multi-monthly rainfall-based drought severity indices, with application to semi-arid Konya closed basin, Turkey. Journal of Hydrology, volumen 470-471, 2012: 255-268.

Elagib N.A. Assessment of drought across central Sudan using UNEP dryness ratio. Hydrology Research, volumen 40 (número 5), 2009: 481-494.

Hargreaves G.H. y Samani Z.A. Estimating Potential Evapotranspiration. Journal of the Irrigation and Drainage Division, volumen 108 (número IR3), 1982: 225-230. 
Machiwal D. y Jha M.K. Comparative evaluation of statistical tests for time series analysis: Applications to hydrological time series. Hydrological Sciences Journal, volumen 53 (número 2), 2008: 353-366.

Machiwal D. y Jha M.K. Hydrologic Time Series Analysis: Theory and Practice, Chapter 4: Methods for Time Series Analysis, pp. 5184, Dordrecht, The Netherlands, Springer, 2012, 303 p.

Mishra A.K. y Singh V.P. A review of drought concepts. Journal of Hydrology, volumen 391 (números 1-2), 2010: 202-216.

Mishra A.K. y Singh V.P. Drought modeling-A review. Journal of Hydrology, volumen 403 (números 1-2), 2011: 157-175.

Morid S., Smakhtin V., Moghaddast M. Comparison of seven meteorological indices for drought monitoring in Iran. International Journal of Climatology, volumen 26 (número 7), 2006: 971-985.

Palmer W.C. Meteorological Drought. Research Paper No. 45, US, Weather Bureau, Washington, USA. 1965, 58 p.

Pandey R.P. y Ramasastri K.S. Incidence of droughts in different climatic regions, Hydrological Science Journal, volumen 47 (número especial), 2002: S31-S40.

Pandey R.P., Sharma K.D., Mishra S.K., Singh R., Agarwal A. Drought Characterization. Chapter 21, pp. 761-792, en: Hydrology and Hydraulics, editado por Vijay P. Singh, Highlands Ranch, Colorado, Water Resources Publications, 2008, 1080 p.

Ponce V.M., Pandey R.P., Ercan S. Characterization of drought across climatic spectrum. Journal of Hydrological Engineering, volumen 5 (número 2), 2000: 222,224.

Tsakiris G., Tigkas D., Vangelis H., Pangalou D. Regional drought identification and assessment. Case study in Crete, Chapter 9, pp. 169-191, en: Methods and tools for drought analysis and mana- gement, editado por Rossi G., Vega T. and Bonaccorso B. Dordrecht, The Netherlands, Springer, 2007, 418 p.

United Nations Environment Programme (UNEP). World Atlas of Desertification, Edward Arnold, Londres, Inglaterra, 1992, 69 plates.

United Nations Environment Programme (UNEP). Global Deserts Outlook. Edited by E. Ezcurra, Division of Early Warning and Assessment, Nairobi, Kenya, 2006, 148 p.

World Meteorological Organization (WMO). Climatic Change. Annexed III: Standard tests of significance to be recommended in routine analysis of climatic fluctuations, pp. 58-71, Technical Note Núm. 79, WMO-Núm. 195, Genova, Suiza, Secretariat of the WMO, 1971, $79 \mathrm{p}$.

\section{Este artículo se cita:}

Citación estilo Chicago

Campos-Aranda, Daniel Francisco. Estudio de sequías meteorológicas anuales por medio del índice de aridez, en el estado de Zacatecas, México. Ingeniería Investigación y Tecnología, XVII, 03 (2016): 405-417.

\section{Citación estilo ISO 690}

Campos-Aranda D.F. Estudio de sequías meteorológicas anuales por medio del índice de aridez, en el estado de Zacatecas, México. Ingeniería Investigación y Tecnología, volumen XVII (número 3), julio-septiembre 2016: 405-417.

\section{Semblanza del autor}

Daniel Francisco Campos-Aranda. Obtuvo el título de ingeniero civil en diciembre de 1972, en la entonces Escuela de Ingeniería de la UASLP. Durante el primer semestre de 1977, realizó en Madrid, España un diplomado en hidrología general y aplicada. Posteriormente, durante 1980-1981 llevó a cabo estudios de maestría en ingeniería en la especialidad de hidráulica, en la División de Estudios de Posgrado de la Facultad de Ingeniería de la UNAM. En esta misma institución, inició (1984) y concluyó (1987) el doctorado en ingeniería con especialidad en aprovechamientos hidráulicos. Ha publicado trabajos principalmente en revistas mexicanas de excelencia: 51 en Tecnología y Ciencias del Agua (antes Ingeniería Hidráulica en México), 19 en Agrociencia y 19 en Ingeniería. Investigación y Tecnología. Es autor de siete textos que versan sobre tópicos de hidrología superficial. En noviembre de 1989 obtuvo la medalla Gabino Barreda de la UNAM y en 2008 le fue otorgado el Premio Nacional "Francisco Torres H." de la AMH. Es profesor jubilado de la UASLP, desde el $1^{\circ}$ de febrero del 2003. A partir de septiembre de 2013 ha vuelto a ser Investigador Nacional nivel I. 